\title{
Suprachoroidal Hemorrhage Simulating Melanoma in Idiopathic Thrombocytopenic Purpura
}

\author{
Stephanie E. Honig Archana Srinivasan Carol L. Shields \\ Ocular Oncology Service, Wills Eye Hospital, Thomas Jefferson University, Philadelphia, PA, USA
}

\section{Established Facts}

- Differentiation of choroidal melanoma from pseudomelanoma is essential but often difficult.

- Ophthalmic involvement in idiopathic thrombocytopenic purpura (ITP) is uncommon, but it is more likely when concomitant anemia and thrombocytopenia are present.

\section{Novel Insights}

- We describe a patient referred with choroidal melanoma but found to have suprachoroidal hemorrhage and underlying ITP.

- A thorough clinical evaluation combined with multimodal imaging is critical to establishing the appropriate diagnosis in eyes with pseudomelanoma.

\section{Keywords}

Choroid · Melanoma · Hemorrhage · Idiopathic thrombocytopenic purpura · Pseudomelanoma

\begin{abstract}
Background: We report a case of suprachoroidal hemorrhage simulating choroidal melanoma in a patient with idiopathic thrombocytopenic purpura (ITP). Methods: Case report. Results: A 79-year-old white male with a history of lung adenocarcinoma, ITP, and mild anemia noted blurred vision with photopsia and floaters in the right eye (OD) that worsened over 2 months. He was found to have a pigment-
\end{abstract}

\section{KARGER}

(c) 2018 S. Karger AG, Basel ed choroidal mass and was referred for evaluation of possible choroidal melanoma. Visual acuity was $20 / 30$ in the affected right eye and 20/25 in the left eye (OS). There was mild vitreous hemorrhage and 2 pigmented peripheral choroidal tumors in the temporal and nasal fundus OD. Bscan ultrasonography confirmed 2 acoustically hollow tumors, and transillumination demonstrated no shadowing. Optical coherence tomography over the lesions revealed choroidal elevation with suprachoroidal scalloped surface. Fluorescein and indocyanine green angiography revealed normal choroidal fluorescence and cyanescence with no "double circulation" of melanoma and no dye blockage, suggesting a suprachoroidal location. These findings were 
suggestive of suprachoroidal hemorrhage rather than melanoma. The patient was managed conservatively, and the hemorrhages demonstrated spontaneous resolution. Conclusion: Ocular manifestations of ITP are uncommon. Rarely, spontaneous suprachoroidal hemorrhage simulating melanoma can occur. A thorough clinical evaluation with multimodal imaging is critical to establishing the appropriate diagnosis.

(c) 2018 S. Karger AG, Basel

\section{Introduction}

Idiopathic thrombocytopenic purpura (ITP) is an autoimmune hemorrhagic condition characterized by accelerated destruction and splenic sequestration of platelets from antiplatelet antibodies [1]. This condition is also termed immune thrombocytopenia and is characterized by a platelet count of less than $100,000 / \mu \mathrm{L}$ and the presence of serum platelet-specific immunoglobulin $\mathrm{G}$ autoantibodies that are generally directed at the most abundant platelet surface glycoproteins (Gp), Gp IIb/IIIa and Gp 1b/IX/V [1].

The incidence of ITP is approximately 3.3 per 100,000 patients per year, and ITP currently affects 9.5 per 100,000 adults in the US [1]. Like most autoimmune conditions, ITP shows a predilection for females, but additionally ITP can be found in young adults and children. The prevalence is fairly equal in older ( $>65$ years of age) males and females $[1,2]$.

ITP is divided into acute, subacute, and chronic types depending on the duration of the disease. In most cases, ITP is a relatively benign disease, commonly presenting with signs of mucocutaneous bleeding, such as easy bruising, petechial and purpuric skin rashes, epistaxis, gingival bleeding, and menorrhagia [1-4]. Patients with mildly decreased platelet counts are often asymptomatic and diagnosed coincidentally, whereas severely affected patients with platelet counts less than $10,000 / \mu \mathrm{L}$ are at risk for serious bleeding complications, particularly intracranial or internal hemorrhagic episodes [4].

Ophthalmic involvement in ITP is uncommon, with few documented cases in the literature [3-11]. Rarely, ITP can cause choroidal hemorrhage or hemorrhagic suprachoroidal effusion mimicking a choroidal melanoma. In a comprehensive analysis of uveal pseudomelanomas, choroidal hemorrhage accounted for only $2 \%$ of cases and uveal effusion for $1 \%$ of cases [12]. Herein, we report a case of hemorrhagic suprachoroidal effusion simulating melanoma in a patient with ITP.

Suprachoroidal Hemorrhage Simulating Melanoma in ITP

\section{Case Report}

A 79-year-old Caucasian male noted blurred vision with photopsia and floaters in the right eye (OD) that worsened over 2 months. He was found to have possible choroidal melanoma and was referred to the Ocular Oncology Service at Wills Eye Hospital, Philadelphia, PA, USA, for evaluation. His past medical history revealed poorly differentiated non-small cell adenocarcinoma of the left lung treated with pneumonectomy and chemotherapy 3 years prior. During the course of lung cancer treatment, he was found to have ITP and was treated with systemic corticosteroids, rituximab, intravenous immunoglobulin (IVIG), and weekly romiplostim, a synthetically manufactured protein specific for immune thrombocytopenia that binds and activates thrombopoietin to increase platelet production. Despite multiple medications, the platelet count remained low at 11,000/ $\mu \mathrm{L}$ (normal 140,000-350,000, ageadjusted mean 249,000), and he required platelet transfusion 2 months before the onset of ophthalmic symptoms that improved the platelet count to $252,000 / \mu \mathrm{L}$. There was no history of trauma to the eye, and the patient also denied recent episodes of excessive coughing, sneezing, vomiting, lifting, or other Valsalva maneuvers.

On our examination, visual acuity was 20/30 OD and 20/25 in the left eye (OS). Intraocular pressure was $16 \mathrm{~mm} \mathrm{Hg}$ OD and 15 $\mathrm{mm} \mathrm{Hg}$ OS. The anterior segment was unremarkable in both eyes. Fundus examination OD revealed hazy ocular media from mild vitreous hemorrhage and 2 pigmented choroidal tumors measuring $10 \mathrm{~mm}$ in diameter temporally and $12 \mathrm{~mm}$ in diameter nasally, both near the ora serrata (Fig. la, b). Fundus examination OS was unremarkable. There were no fundus photographs from the referring ophthalmologist for comparison.

B-scan ultrasonography OD confirmed 2 acoustically hollow tumors measuring $4.0 \mathrm{~mm}$ in thickness temporally and $3.1 \mathrm{~mm}$ nasally (Fig. 1g). Transillumination demonstrated no shadowing, suggestive of a nonmelanoma condition. Optical coherence tomography (OCT) of the peripheral lesions revealed choroidal elevation with a scalloped surface, extending from the periphery into the submacular region (Fig. 1e, f). Fluorescein angiography (FA) and indocyanine green angiography (ICGA) revealed normal choroidal fluorescence with no "double circulation" of melanoma and no blockage of fluorescence or cyanescence, implying that the pigmented lesions were located in the suprachoroidal space (Fig. 1c, d). These findings were suggestive of suprachoroidal hemorrhage rather than choroidal melanoma.

Given the history of ITP and recent thrombocytopenia requiring transfusion, the suprachoroidal hemorrhage was managed with conservative observation for hemorrhage resolution. On follow-up 1 month later, visual acuity was 20/50 OD and 20/25 OS. Fundus examination revealed resolving hemorrhage with decreased thickness to $3.1 \mathrm{~mm}$ temporally and $2.3 \mathrm{~mm}$ nasally. Conservative management was continued.

\section{Discussion}

Differentiation of choroidal melanoma from pseudomelanoma is essential but often difficult. Choroidal melanoma typically presents as a painless, elevated, dome or mushroom-shaped pigmented mass often associated

Ocul Oncol Pathol 2019;5:162-166 
Fig. 1. A 79-year-old Caucasian male with idiopathic thrombocytopenic purpura and sudden photopsia was found to have possible choroidal melanoma. a Fundus photograph showing mild vitreous hemorrhage and 2 darkly pigmented choroidal tumors (arrows) in the nasal and temporal quadrants of the right eye. b Wide-angle Heidelberg image reveals presence of hemorrhagic choroidal effusion (arrows). c, d Fluorescein angiography and indocyanine green angiography reveal normal choroidal vasculature without "double circulation" of melanoma and with minor vitreous hemorrhage blockage. e, f Optical coherence tomography demonstrates extensive, shallow choroidal folds with abrupt elevation of the retina and retinal pigment epithelium. g B-scan ultrasonography shows acoustically empty mass measuring $4.0 \mathrm{~mm}$ in thickness.
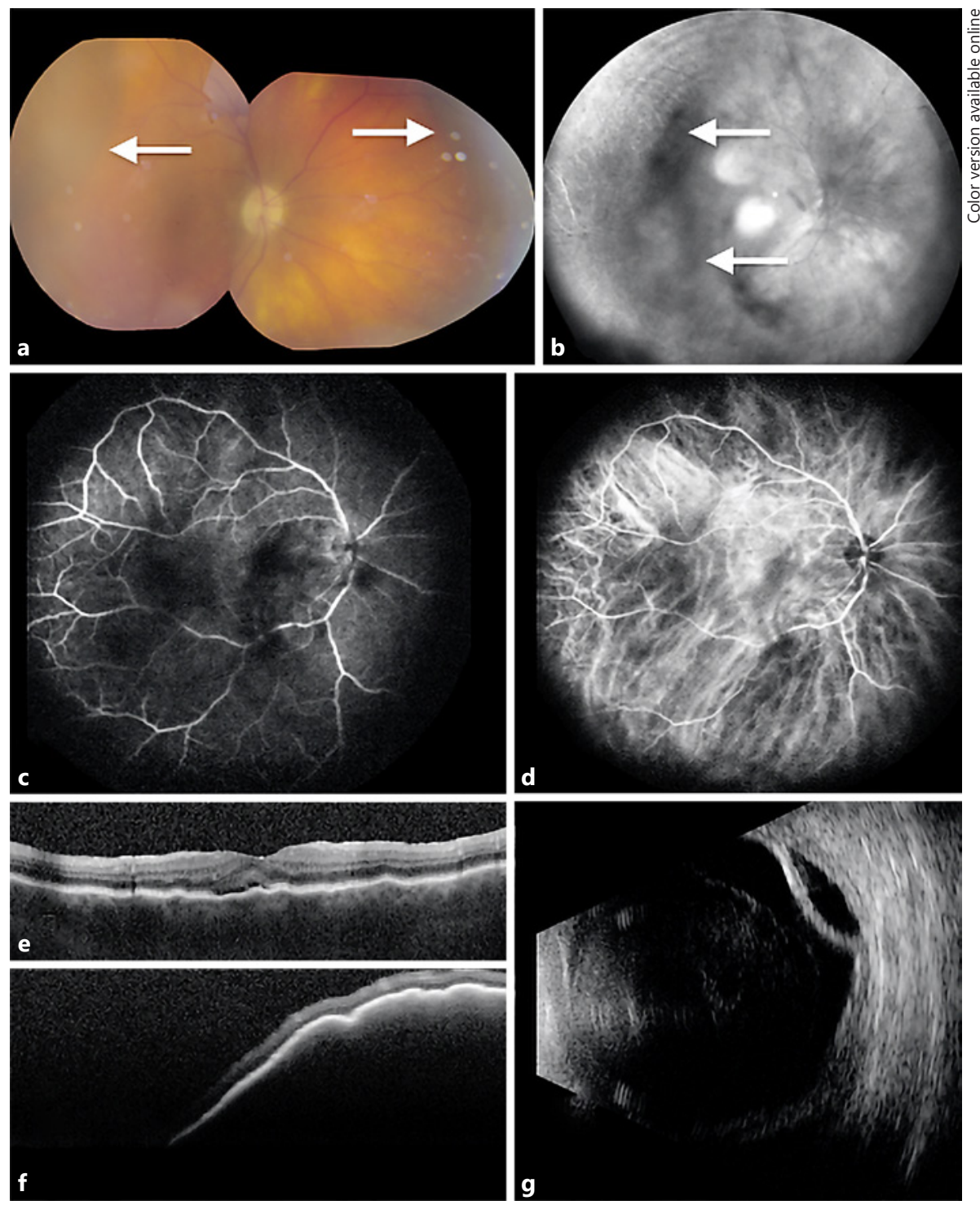

with subretinal fluid and orange pigment [13]. Several conditions can simulate choroidal melanoma, leading to incorrect diagnosis. In a review of over 12,000 patients referred to a tertiary referral center for management of choroidal melanoma, 1,739 (14\%) were simulating conditions [12]. The most common etiologies included choroidal nevus (49\%), peripheral exudative hemorrhagic chorioretinopathy (8\%), congenital hypertrophy of the retinal pigment epithelium (6\%), hemorrhagic detachment of the retina or retinal pigment epithelium (5\%), circumscribed choroidal hemangioma (5\%), and age-related macular degeneration/disciform scar (4\%). Hemorrhagic choroidal detachment accounted for only $2 \%$ of pseudomelanomas [12].
Both choroidal melanoma and hemorrhagic choroidal detachment (also known as suprachoroidal hemorrhage) can manifest as a brown-red, dome-shaped mass appearing in the choroid. However, B-scan ultrasonography, FA or ICGA, and OCT assist in differentiating the 2 entities $[13,14]$. On B-scan ultrasonography, choroidal melanoma is generally acoustically hollow, compared to suprachoroidal hemorrhage that can be either acoustically hollow or solid, depending on the amount of blood [12-14]. On FA, choroidal melanoma shows "double circulation" with vessels residing in the solid mass, whereas choroidal hemorrhage shows no increased vascularity. Similarly, on ICGA, choroidal melanoma demonstrates intrinsic vascularity, whereas suprachoroidal 
hemorrhage is usually silent as it occurs external to the choroidal vasculature [14]. On OCT, choroidal melanoma typically shows a smooth tumor surface with overlying choriocapillaris compression, pronounced optical shadowing, and subretinal fluid in some cases. In contrast, suprachoroidal hemorrhage shows an elevated choroid with an often scalloped surface to the hemorrhage that is located external to the choroid [14]. As the suprachoroidal hemorrhage resolves, the OCT documents tumor reduction with choroidal folds and ultimately flat choroid.

ITP is an autoimmune disease characterized by autoantibodies in about $60 \%$ of cases [1]. The most common platelet antigens are Gp IIb/IIIa and Gp Ib/IX/V. T cells have also been implicated in the pathogenesis of ITP [1]. ITP can be primary or secondary based on the presence or absence of underlying diseases, such as systemic lupus erythematosus, lymphoproliferative disorders, chronic infections like Helicobacter pylori or HIV, or drug exposure, such as quinine or heparin [15]. The clinical symptoms and presentations vary depending on the severity of the diminished platelet count [1]. Treatment options for ITP include observation, systemic immunosuppressive therapy (corticosteroids, IVIG, or rituximab), splenectomy, and thrombopoietin receptor agonists, such as romiplostim $[1,2]$.

Ocular manifestations of ITP are uncommon and vary widely, ranging from benign findings, such as chemosis, subconjunctival hemorrhage, and hematoma causing corneal surface irregularities or bloody tears, to more significant pathology, including retinal, vitreous, peripapillary, or subhyaloid hemorrhage, optic tract hematoma, and ischemic optic neuropathy [3,6-11]. In a survey of 85 patients with chronic ITP by Matzdorff and Arnold [16], 11\% had either intraocular hemorrhage with visual impairment or intracerebral hemorrhage. Severe vision loss can result from subretinal and intraretinal hemorrhage and residual serous retinal detachment following resorption of blood [3]. Thrombocytopenia alone rarely causes significant retinal or subretinal hemorrhage, but additional anemia can spawn hemorrhages $[8,14]$. Shah et al. [3] described bilateral subretinal and intraretinal hemorrhage associated with ITP in a previously healthy 26-year-old patient who subsequently developed an intracranial hemorrhage that required emergency neurosurgical intervention.

The finding of choroidal hemorrhage simulating melanoma has been documented by Shields et al. [12] in 2005 and Fung et al. [14] in 2013. Marous et al. [17] have also described 2 cases of coughing-induced supracho- roidal hemorrhage simulating melanoma. However, development of suprachoroidal hemorrhage in a patient with ITP has rarely been recognized. Al-Debasi et al. [18] reported a 13-year-old child with ITP who developed spontaneous suprachoroidal hemorrhage that led to total choroidal detachment and was treated with systemic corticosteroids and IVIG to improve the platelet count. Later, surgical drainage of the suprachoroidal hemorrhage was performed after platelet count stabilization. Our case is unique in that the spontaneous suprachoroidal hemorrhage occurred in an adult patient with ITP, simulating melanoma. Our patient had a combination of ITP and anemia, with an initial platelet count of $11,000 / \mu \mathrm{L}$ and hemoglobin level of $11.1 \mathrm{~g} / \mathrm{dL}$ (normal 12.5-17.0).

In summary, multimodal imaging is essential in distinguishing choroidal melanoma from simulating choroidal lesions. In our case, imaging with OCT as well as IVFA and ICG and careful history-taking led to the diagnosis of hemorrhage and not melanoma. Although uncommon, suprachoroidal hemorrhage can simulate choroidal melanoma, especially in patients with hematologic abnormalities, such as ITP and anemia.

\section{Statement of Ethics}

Our case report complies with all guidelines for human studies and animal welfare regulations. The patient has given informed consent. Our Institute's committee on human research has approved the study's protocol. Our case study does not include any animal experimentation.

\section{Disclosure Statement}

No conflicting relationship exists for any author.

\section{Funding Sources}

Support was provided by the Eye Tumor Research Foundation, Philadelphia, PA (C.L.S.). The funders had no role in the design and conduct of the study, in the collection, analysis, and interpretation of the data, or in the preparation, review, or approval of the manuscript. Carol L. Shields, MD, has had full access to all the data in the study and takes responsibility for the integrity of the data and the accuracy of the data analysis.

Ocul Oncol Pathol 2019;5:162-166

DOI: $10.1159 / 000490390$
Suprachoroidal Hemorrhage Simulating

Melanoma in ITP 


\section{References}

1 Lambert MP, Gernsheimer TB. Clinical updates in adult immune thrombocytopenia. Blood. 2017 May;129(21):2829-35.

2 Nomura S. Advances in diagnosis and treatments for immune thrombocytopenia. Clin Med Insights Blood Disord. 2016 Jul;9:15-22.

3 Shah PA, Yang SS, Fung WE. Idiopathic thrombocytopenic purpura with massive subretinal hemorrhage. Arch Ophthalmol. 2005 Nov;123(11):1612-3.

4 Schoonen WM, Kucera G, Coalson J, Li L, Rutstein M, Mowat F, et al. Epidemiology of immune thrombocytopenic purpura in the General Practice Research Database. Br J Haematol. 2009 Apr; 145(2):235-44.

5 Sodhi PK, Jose R. Subconjunctival hemorrhage: the first presenting clinical feature of idiopathic thrombocytopenic purpura. Jpn J Ophthalmol. 2003 May-Jun;47(3):316-8.

6 Turaka K, Shields CL, Bianciotto C, Shields JA. Vitreous hemorrhage as the initial manifestation of idiopathic thrombocytopenic purpura. Retin Cases Brief Rep. 2012;6(1):168.

7 Goel N, Arora S, Jain P, Ghosh B. Massive subretinal and vitreous haemorrhages at presentation in idiopathic thrombocytopenic purpura: report of a case and review of literature. Clin Exp Optom. 2014 May;97(3):270-3.
8 Rubenstein RA, Yanoff M, Albert DM. Thrombocytopenia, anemia, and retinal hemorrhage. Am J Ophthalmol. 1968 Mar;65(3): 435-9.

9 Wan-Wei L, Tengku-Norina TJ, Azma-Azalina AA, Zulkifli AG, Zunaina E. Spontaneous bilateral peripapillary, subhyaloid and vitreous hemorrhage with only minor platelet deficit in idiopathic thrombocytopenic purpura. Int Med Case Rep J. 2014 Jan;7:15-7.

10 Inoue T, Yanagi Y, Tamaki Y, Kami J, Kato Y. Massive subretinal haemorrhage secondary to age-related macular degeneration in a patient with idiopathic thrombocytopenic purpura. Eye (Lond). 2004 Jun;18(6):656-7.

11 Carraro MC, Rossetti L, Gerli GC. Prevalence of retinopathy in patients with anemia or thrombocytopenia. Eur J Haematol. 2001 Oct; 67(4):238-44.

12 Shields JA, Mashayekhi A, Ra S, Shields CL. Pseudomelanomas of the posterior uveal tract: the 2006 Taylor R. Smith Lecture. Retina. 2005 Sep;25(6):767-71.
13 Shields JA, Shields CL. Posterior uveal melanoma: Diagnostic approaches. In: Shields JA, Shields CL, editors. Intraocular Tumors: An Atlas and Textbook. 3rd ed. Philadelphia (PA): Lippincott, Wolters Kluwers; 2016. pp. 139-53.

14 Fung AT, Fulco EM, Shields CL, Shields JA. Choroidal hemorrhage simulating choroidal melanoma. Retina. 2013 Sep;33(8):1726-8.

15 Rodeghiero F, Stasi R, Gernsheimer T, Michel M, Provan D, Arnold DM, et al. Standardization of terminology, definitions and outcome criteria in immune thrombocytopenic purpura of adults and children: report from an international working group. Blood. 2009 Mar; 113(11):2386-93

16 Matzdorff A, Arnold G. Treatment of chronic immune thrombocytopenic purpura: the patients' perspective. Eur J Haematol. 2007 May; 78(5):381-8.

17 Marous CL, Sioufi K, Shields CL, Mashayekhi A, Shields JA. Coughing-induced suprachoroidal hemorrhage simulating melanoma in two cases. Retin Cases Brief Rep. 2016 Nov; $0(0): 1-6$

18 Al-Debasi T, Khuthaila MK, Al-Fakhri AS, Al-Ferayan YA, Bashir R, Abbasi A, et al. Spontaneous suprachoroidal hemorrhage in a thirteen-year-old child with thrombocytopenic purpura. Am J Case Rep. 2017;5(6):156-9. 